\title{
High-dose daptomycin and fosfomycin treatment of a patient with endocarditis caused by daptomycin-nonsusceptible Staphylococcus aureus: Case report
}

Liang-Yu Chen ${ }^{1,3}$, Cheng-Hsiung Huang ${ }^{2,3}$, Shu-Chen Kuo ${ }^{1,3}$, Chen-Yuan Hsiao ${ }^{2}$, Mei-Lin Lin ${ }^{1}$, Fu-Der Wang ${ }^{1,3^{*}}$ and Chang-Phone Fung ${ }^{1,3}$

\begin{abstract}
Background: Emergence of daptomycin-nonsusceptible (DNS) Staphylococcus aureus is a dreadful problem in the treatment of endocarditis. Few current therapeutic agents are effective for treating infections caused by DNS $S$. aureus.

Case presentation: We describe the emergence of DNS S. aureus. in a patient with implantable cardioverterdefibrillator (ICD) device -related endocarditis who was priorily treated with daptomycin. Metastatic dissemination as osteomyelitis further complicated the management of endocarditis. The dilemma was successfully managed by surgical removal of the ICD device and combination antimicrobial therapy with high-dose daptomycin and fosfomycin.

Conclusions: Surgical removal of intracardiac devices remains an important adjunctive measure in the treatment of endocarditis. Our case suggests that combination therapy is more favorable than single-agent therapy for infections caused by DNS S. aureus.
\end{abstract}

Keywords: Daptomycin-nonsusceptible, fosfomycin, ICD device-related endocarditis

\section{Background}

Infective endocarditis is a clinically significant disease with mortality ranging from $16 \%$ to $25 \%[1,2]$, and Staphylococcus aureus is reported as the most common pathogen, accounting for $31.4 \%$ of cases in developing countries [3]. In addition to methicillin-resistant S. aureus (MRSA), the emergence of vancomycin-resistant $S$. aureus also became a major problem after the first report in Japan in 1997 [4].

Daptomycin is a 13 -aminoacid compound derived from fermentation of Streptomyces roseosporus and is a new treatment option for $S$. aureus endocarditis, including vancomycin-resistant $S$. aureus [5,6]. The once daily dosing of daptomycin $6 \mathrm{mg} / \mathrm{kg}$ approved by the USA

\footnotetext{
* Correspondence: fdwang@vghtpe.gov.tw

'Division of Infectious Diseases, Department of Medicine, Taipei Veterans General Hospital, Taipei, Taiwan

Full list of author information is available at the end of the article
}

Food and Drug administration showed non-inferiority to the standard therapy for treating $S$. aureus bacteremia and endocarditis [7]. However, increased incidence of daptomycin-nonsusceptible (DNS) S. aureus was reported recently. Mutations in the $\operatorname{mprF}$ and $y y c F G$ genes, related to membrane fluidity changes or fatty acid biosynthesis, were identified recently as possible contributors to daptomycin nonsusceptibility $[5,8]$. Effective management in case of DNS S. aureus infections is an important issue nowadays.

Fosfomycin, a phosphonic acid derivative (cis-1,2epoxypropyl phosphonic acid), was initially described and isolated in 1969 from cultures of Streptomyces species [9]. It was commonly employed in treating urinary tract infections and has a bactericidal mechanism of action $[9,10]$. Because it is a relatively small, hydrophilic molecule with almost negligible serum protein binding and good tissue penetration, this agent was more
C Biomed Central

(c) 2011 Chen et al; licensee BioMed Central Ltd. This is an Open Access article distributed under the terms of the Creative Commons Attribution License (http://creativecommons.org/licenses/by/2.0), which permits unrestricted use, distribution, and reproduction in any medium, provided the original work is properly cited. 
frequently used in combination therapy for treating multidrug-resistant pathogens $[10,11]$.

We present our treatment experience utilizing surgical intervention and high-dose daptomycin in combination with fosfomycin for successful treatment of a patient with implantable cardioverter-defibrillator (ICD) devicerelated endocarditis complicated with osteomyelitis caused by DNS MRSA.

\section{Case Presentation}

A 28-year-old woman had a history of sudden onset of syncope diagnosed as paroxysmal ventricular tachycardia. She underwent electric ablation and placement of an ICD in her left shoulder. She had poor wound healing at a 5-month follow-up visit and she was admitted to the hospital on December 1, 2008 for management of a pocket infection. Exposure of the ICD generator and pacing lead were found on February 26, 2009, and the generator was removed, leaving the pacing lead in place. She had episodic pyrexia beginning in May 2009 and her blood cultures grew MRSA (isolate st01, positive in one of two sets blood cultures collected). ICD devicerelated endocarditis was highly suspected. Intravenous antibiotics with vancomycin $1 \mathrm{~g}$ every 12 hours for 14 days followed by oral linezolid $600 \mathrm{mg}$ every 12 hours for another 14 days were prescribed for her endocarditis, but the blood cultures still grew MRSA (isolate st02, positive in one of two sets blood cultures collected). A thoracotomy was planned to remove the pacing lead. However, because of three episodes of broken peripheral venous catheter tips left in the vessels, generator displacement requiring surgical intervention, and emotional fragility, the surgical intervention was postponed due to the high possibility of postoperative wound infection and further osteomyelitis. Daptomycin at a dosage of 6 $\mathrm{mg} / \mathrm{kg}$ daily was prescribed for its effective biofilm penetration ability and rapid bacteriocidal efficacy, and the subsequent blood culture (one set collected) yielded negative finding. A total 65 days of treatment were completed and the patient was discharged on October 24, 2009.

Unfortunately, spiking fever and chills occurred 1 day after discharge and the blood cultures grew MRSA again (isolates st03, positive in one of two blood cultures collected). During antibiotic treatment with intravenous tigecycline (100-mg loading dose and $50 \mathrm{mg}$ every 12 hours for 14 days), one of two sets of blood cultures grew MRSA (isolate st04). Teicoplanin $400 \mathrm{mg}$ every 12 hours for another 14 days was given, but another one of two sets of blood cultures grew MRSA (isolate st05). Intravenous daptomycin was prescribed at a dosage of up to $9 \mathrm{mg} / \mathrm{kg}$ daily in combination with intravenous fosfomycin 6 g every 6 hours starting December 16, 2009, due to the persistence of MRSA bacteremia with the ICD device in place. The patient's fever subsided after these treatments and the next three sets of blood cultures were negative 1 week after the start of daptomycin-fosfomycin combination therapy. However, transesophageal echocardiography revealed vegetations growing on the pacing lead, and a scan for osteomyelitis showed increased uptake of the radiotracer in the left proximal clavicle. Moreover, the daptomycin Etest (AB Biodisk, Solna, Sweden) of MRSA isolate st05 indicated that it was nonsusceptible to daptomycin, with a minimal inhibitory concentration (MIC) ranging from 1.5 to $2 \mathrm{mg} / \mathrm{L}$. After discussion among the surgeons and infectious disease specialists, a thoracotomy was performed on December 22, 2009 for removal of the pacing lead due to the previous persistence of $S$. aureus bacteremia; high-dose daptomycin $12 \mathrm{mg} / \mathrm{kg}$ intravenously daily was also started. A vegetation measuring $1.3 \times 0.6 \times 0.1 \mathrm{~cm}$ was found on the pacing lead, but the Gram stain showed no microbes. Tissue culture of the vegetation found on the pacing lead grew MRSA (isolate st06), as did as the tip of the pacing lead (isolate st07).

Because the osteomyelitis scan was positive, combination therapy with daptomycin $12 \mathrm{mg} / \mathrm{kg}$ intravenously daily and fosfomycin $6 \mathrm{~g}$ intravenously every 6 hours was administered for total of 56 days. The patient had no further pyretic episodes following hospitalization. A follow-up osteomyelitis scan on March 8, 2010 was negative, and follow-up transthoracic echocardiography showed no vegetation. The patient's symptoms were attributed to endocarditis; she remained symptom free during 12 months of follow-up.

\section{Methods}

The species identification was completed using the API 32GN biochemical testing system (bioMérieux, Inc., Marcy l'Étoile, France). Antimicrobial susceptibility testing was performed using agar dilution method with 25 $\mathrm{mg} / \mathrm{L}$ glucose- 6 phosphate added for fosfomycin testing; the broth dilution method of antimicrobial susceptibility testing was used for the other antimicrobial agents, as specified by the Clinical Laboratory Standards Institute (CLSI 2010), using the VITEK ${ }^{\circledR} 2$ Compact system (bioMérieux, Inc.,) and daptomycin Etest strips (AB Biodisk, Solna, Sweden). Guidance for interpreting the MICs as clinical categories (susceptible, intermediate, or resistant) was provided by the manufacturer's instructions and CLSI criteria. Isolates with daptomycin MICs $\leq 1$ $\mathrm{mg} / \mathrm{L}$ were defined as daptomycin-susceptible, and those with MICs $>1 \mathrm{mg} / \mathrm{L}$ were defined as daptomycin-nonsusceptible. No MIC interpretation criteria were available for fosfomycin on bacteremia in CLSI criteria, but isolates with MICs less than $32 \mathrm{mg} / \mathrm{L}$ were defined as susceptible by the European Committee on Antimicrobial Susceptibility Testing (EUCAST). In order to 
establish the clonality of these isolates, we performed pulsed-field gel electrophoresis (PFGE) as described previously [12]. S. aureus ATCC 29213 was used as the quality control organism.

\section{Results}

The seven isolates were analyzed by their Sma1 PFGE profiles and isolates st02 to st06 showed the same profile, while isolates st01 and st07 were different. Progressive elevation of the daptomycin MICs for isolates of the same clonality occurred, but decreasing MICs for isolate st06 were noted for vancomycin, teicoplanin, and daptomycin compared to those of isolate st05 (Table 1). The MICs of fosfomycin for all isolates were less than 2 $\mathrm{mg} / \mathrm{L}$ by the agar dilution methods and showed susceptibility based on the EUCAST criteria. All isolates were resistant to the other antimicrobials screened using the VITEK $^{\circledR} 2$ Compact system (Table 1 ).

\section{Conclusions}

Combination therapy consisting of high-dose daptomycin and fosfomycin is an alternative treatment option for DNS MRSA native valve endocarditis or osteomyelitis. These two agents are well known for their bacteriocidal activities and effective biofilm penetration $[5,6,10,11]$. Recently, positive results for fosfomycin used in combination with other antimicrobial agents against multidrug resistant Gram-positive pathogens were also reported $[9,11]$.

Isolate st06 from the vegetation, of the same clonality as isolate st05, was susceptible to vancomycin, teicoplanin, and daptomycin in combination with fosfomycin with additive effect. Additionally, the pathological findings of the vegetation were negative for microbes, which suggested that the combination regimen was highly effective in biofilm penetration and killing the bacteria. Moreover, the osteomyelitis was successfully treated in 8 weeks, without complications. Although high-dose daptomycin treatment could be effective after surgical removal of an ICDdevice for adequate pharmacodynamics attainments, the combination therapy is preferred in complicated cases, and further study to establish the adequate combination dosage of daptomycin with fosfomycin is needed. Steed et al. also reported that the combination of daptomycin plus clotrimoxazole was bactericidal against DNS MRSA in an in vitro model of simulated endocardial vegetations [13].

High-dose daptomycin treatment ( $12 \mathrm{mg} / \mathrm{kg}$ daily) was well tolerated in our patient throughout the 8-week treatment course. In a previous study, patients received daptomycin in dosages greater than $8 \mathrm{mg} / \mathrm{kg}$ per day, and $79 \%$ of patients were considered evaluable for efficacy; only $6.4 \%$ experienced 1 or more adverse events or abnormal laboratory analyte values [14]. Another recent report also revealed no remarkable creatinine kinase elevation after daptomycin use in dosage greater than 8 $\mathrm{mg} / \mathrm{kg}$ once daily for a prolonged course of 14 days [15]. Because the resistance mechanism of $S$. aureus to daptomycin is unique in comparison to other glycopeptides, and every single gene mutation, such as $m p r F$ and $y y c F G$ genes, causes only a slight increase in daptomycin MIC, higher doses of daptomycin could still be effective for the treatment of DNS S. aureus [5,8]. Nonetheless, in vitro and in vivo evidence suggests that combination therapy is more favorable than single-agent therapy for infections caused by DNS S. aureus, and antibiotic therapy must be individualized for each patient's condition.

For patients with ICD device-related endocarditis, surgical removal of the device is strongly recommended when $S$. aureus bacteremia persists after antimicrobial therapy $[16,17]$. As reported by del Rio A et al. the prevalence of treatment failure is greater among patients receiving only antibiotics therapy compared to those treated with surgical removal of implanted device and antibiotics therapy [18]. Recurrence of MRSA bacteremia identified during our patient's second visit suggested treatment failure, even though the blood cultures were negative after the initial daptomycin treatment; such patients should undergo prompt surgical removal of the ICD device. It is possible that with daptomycin alone the patient could have been cured in the first attempt if

Table 1 Antimicrobial susceptibility results for the seven methicillin-resistant Staphylococcus aureus clinical isolates

\begin{tabular}{|c|c|c|c|c|c|c|c|c|c|}
\hline \multirow[t]{3}{*}{ Isolate } & \multirow[t]{3}{*}{ Date of isolation } & \multicolumn{8}{|c|}{ MIC (mg/L) } \\
\hline & & \multicolumn{3}{|c|}{ Vitek 2} & \multicolumn{3}{|c|}{ Broth dilution } & \multirow{2}{*}{$\begin{array}{c}\text { Agar dilution } \\
\text { FOS }\end{array}$} & \multirow{2}{*}{$\begin{array}{l}\text { Etest } \\
\text { DPC }\end{array}$} \\
\hline & & VAN & TEIC & LIN & VAN & TEIC & DPC & & \\
\hline st01 & 2009/5/19 & $<0.5$ & $<0.5$ & 2 & 1 & 1 & 0.25 & $<2$ & 0.19 \\
\hline st02 & 2009/9/27 & 2 & $<0.5$ & 2 & 1 & 1 & 0.25 & $<2$ & 0.38 \\
\hline st03 & 2009/10/29 & 2 & $<0.5$ & 4 & 1 & 1 & 0.5 & $<2$ & 0.25 \\
\hline st04 & 2009/11/10 & 1 & $<0.5$ & 4 & 1 & 1 & 0.5 & $<2$ & 0.25 \\
\hline st05 & 2009/12/11 & 2 & 2 & 4 & 2 & 0.25 & 1 & $<2$ & $1.5-2$ \\
\hline st06 & 2009/12/22 & 2 & $<0.5$ & 2 & 1 & 0.5 & 0.5 & $<2$ & 0.25 \\
\hline st07 & $2009 / 12 / 22$ & 2 & 1 & 4 & 2 & 4 & 2 & $<2$ & 1 \\
\hline
\end{tabular}

DPC: daptomycin; FOS: fosfomycin; LIN: linezolid; MIC: minimal inhibitory concentration; TEIC: teicoplanin; VAN: vancomycin 
the ICD lead had been surgical removed. Although the combination therapy achieved negative blood cultures, the tissue culture of the vegetation and pacing lead still grew MRSA, with isolate st07 showing increased daptomycin resistance. Recurrence would have remained possible without the removal of the pacing lead.

\section{Consent}

Written informed consent was obtained from the patient for publication of this case report.

\section{Acknowledgements}

We thank Mr. Chi-Chung Chen (E-mail address: ccomm2@yahoo.com.tw) from Department of Internal Medicine, Medical Research, Chi Mei Medical Center, Tainan, Taiwan, for his technique support on antimicrobial susceptibility tests.

\section{Author details}

${ }^{1}$ Division of Infectious Diseases, Department of Medicine, Taipei Veterans General Hospital, Taipei, Taiwan. ²Division of Cardiovascular Surgery, Department of Surgery, Taipei Veterans General Hospital, Taipei, Taiwan. ${ }^{3}$ School of Medicine, National Yang-Ming University, Taipei, Taiwan.

\section{Authors' contributions}

LYC, SCK, and MLL completed the microbial cultures, agar and broth microdilutions for MICs and PFGE. $\mathrm{CHH}$ and $\mathrm{CYH}$ took responsibility of the patient on admission for wound debridement, thoracotomy, and postsurgical care. FDW and CPF suggested and decided which antibiotics were administered and suggested the plan of action based on the advanced image examinations. All authors read and approved the manuscript.

\section{Competing interests}

The authors declare that they have no competing interests.

Received: 26 October 2010 Accepted: 26 May 2011

Published: 26 May 2011

\section{References}

1. Moreillon P, Que YA: Infective endocarditis. Lancet 2004, 363:139-149.

2. Mylonakis E, Calderwood SB: Infective endocarditis in adults. N Engl J Med 2001, 345:1318-1330.

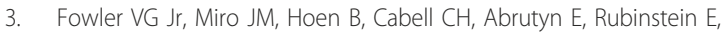
Corey GR, Spelman D, Bradley SF, Barsic B, et al: Staphylococcus aureus endocarditis: a consequence of medical progress. JAMA 2005, 293:3012-3021.

4. Hiramatsu K, Hanaki H, Ino T, Yabuta K, Oguri T, Tenover FC: Methicillinresistant Staphylococcus aureus clinical strain with reduced vancomycin susceptibility. J Antimicrob Chemother 1997, 40:135-136.

5. Baltz RH: Daptomycin: mechanisms of action and resistance, and biosynthetic engineering. Curr Opin Chem Biol 2009, 13:144-151.

6. Falagas ME, Giannopoulou KP, Ntziora F, Vardakas KZ: Daptomycin for endocarditis and/or bacteraemia: a systematic review of the experimental and clinical evidence. J Antimicrob Chemother 2007, 60:7-19.

7. Fowler VG Jr, Boucher HW, Corey GR, Abrutyn E, Karchmer AW, Rupp ME, Levine DP, Chambers HF, Tally FP, Vigliani GA, et al: Daptomycin versus standard therapy for bacteremia and endocarditis caused by Staphylococcus aureus. N Engl J Med 2006, 355:653-665.

8. Jones T, Yeaman MR, Sakoulas G, Yang SJ, Proctor RA, Sahl HG, Schrenzel J, Xiong YQ, Bayer AS: Failures in clinical treatment of Staphylococcus aureus Infection with daptomycin are associated with alterations in surface charge, membrane phospholipid asymmetry, and drug binding. Antimicrob Agents Chemother 2008, 52:269-278.

9. Falagas ME, Giannopoulou KP, Kokolakis GN, Rafailidis PI: Fosfomycin: use beyond urinary tract and gastrointestinal infections. Clin Infect Dis 2008, 46:1069-1077.
10. Roussos N, Karageorgopoulos DE, Samonis G, Falagas ME: Clinical significance of the pharmacokinetic and pharmacodynamic characteristics of fosfomycin for the treatment of patients with systemic infections. Int J Antimicrob Agents 2009, 34:506-515.

11. Falagas ME, Roussos N, Gkegkes ID, Rafailidis PI, Karageorgopoulos DE: Fosfomycin for the treatment of infections caused by Gram-positive cocci with advanced antimicrobial drug resistance: a review of microbiological, animal and clinical studies. Expert Opin Investig Drugs 2009, 18:921-944.

12. Murchan S, Kaufmann ME, Deplano A, de Ryck R, Struelens M, Zinn CE, Fussing V, Salmenlinna S, Vuopio-Varkila J, El Solh N, et al: Harmonization of pulsed-field gel electrophoresis protocols for epidemiological typing of strains of methicillin-resistant Staphylococcus aureus: a single approach developed by consensus in 10 European laboratories and its application for tracing the spread of related strains. J Clin Microbiol 2003, 41:1574-1585.

13. Steed ME, Vidaillac C, Rybak MJ: Novel daptomycin combinations against daptomycin-nonsusceptible methicillin-resistant Staphylococcus aureus in an in vitro model of simulated endocardial vegetations. Antimicrob Agents Chemother 2010, 54:5187-5192.

14. Moise PA, Hershberger E, Amodio-Groton MI, Lamp KC: Safety and clinical outcomes when utilizing high-dose (> or $=8 \mathrm{mg} / \mathrm{kg}$ ) daptomycin therapy. Ann Pharmacother 2009, 43:1211-1219.

15. Figueroa DA, Mangini E, Amodio-Groton M, Vardianos B, Melchert A, Fana C, Wehbeh W, Urban CM, Segal-Maurer S: Safety of high-dose intravenous daptomycin treatment: three-year cumulative experience in a clinical program. Clin Infect Dis 2009, 49:177-180.

16. Baddour LM, Epstein AE, Erickson CC, Knight BP, Levison ME, Lockhart PB, Masoudi FA, Okum EJ, Wilson WR, Beerman LB, et al: Update on cardiovascular implantable electronic device infections and their management: a scientific statement from the American Heart Association. Circulation 2010, 121:458-477.

17. Baddour LM, Wilson WR, Bayer AS, Fowler VG Jr, Bolger AF, Levison ME, Ferrieri P, Gerber MA, Tani LY, Gewitz MH, et al: Infective endocarditis: diagnosis, antimicrobial therapy, and management of complications: a statement for healthcare professionals from the Committee on Rheumatic Fever, Endocarditis, and Kawasaki Disease, Council on Cardiovascular Disease in the Young, and the Councils on Clinical Cardiology, Stroke, and Cardiovascular Surgery and Anesthesia, American Heart Association: endorsed by the Infectious Diseases Society of America. Circulation 2005, 111:e394-434.

18. del Rio A, Anguera I, Miro JM, Mont L, Fowler VG Jr, Azqueta M, Mestres CA: Surgical treatment of pacemaker and defibrillator lead endocarditis: the impact of electrode lead extraction on outcome. Chest 2003, 124:1451-1459.

\section{Pre-publication history}

The pre-publication history for this paper can be accessed here: http://www.biomedcentral.com/1471-2334/11/152/prepub

doi:10.1186/1471-2334-11-152

Cite this article as: Chen et al:: High-dose daptomycin and fosfomycin treatment of a patient with endocarditis caused by daptomycinnonsusceptible Staphylococcus aureus: Case report. BMC Infectious Diseases 2011 11:152.

\section{Submit your next manuscript to BioMed Central and take full advantage of:}

- Convenient online submission

- Thorough peer review

- No space constraints or color figure charges

- Immediate publication on acceptance

- Inclusion in PubMed, CAS, Scopus and Google Scholar

- Research which is freely available for redistribution 